\title{
Correction to: Pathologic Basis for Thyroid Ultrasound
}

\author{
Grace C. H. Yang
}

\section{Correction to: \\ K. Kakudo (ed.), Thyroid FNA Cytology, https://doi.org/10.1007/978-981-13-1897-9}

In the original version of Fig. 20.2 (Chapter 20), part labels were incorrectly placed. The corrected version of the figure has been updated in the book and it is presented below.
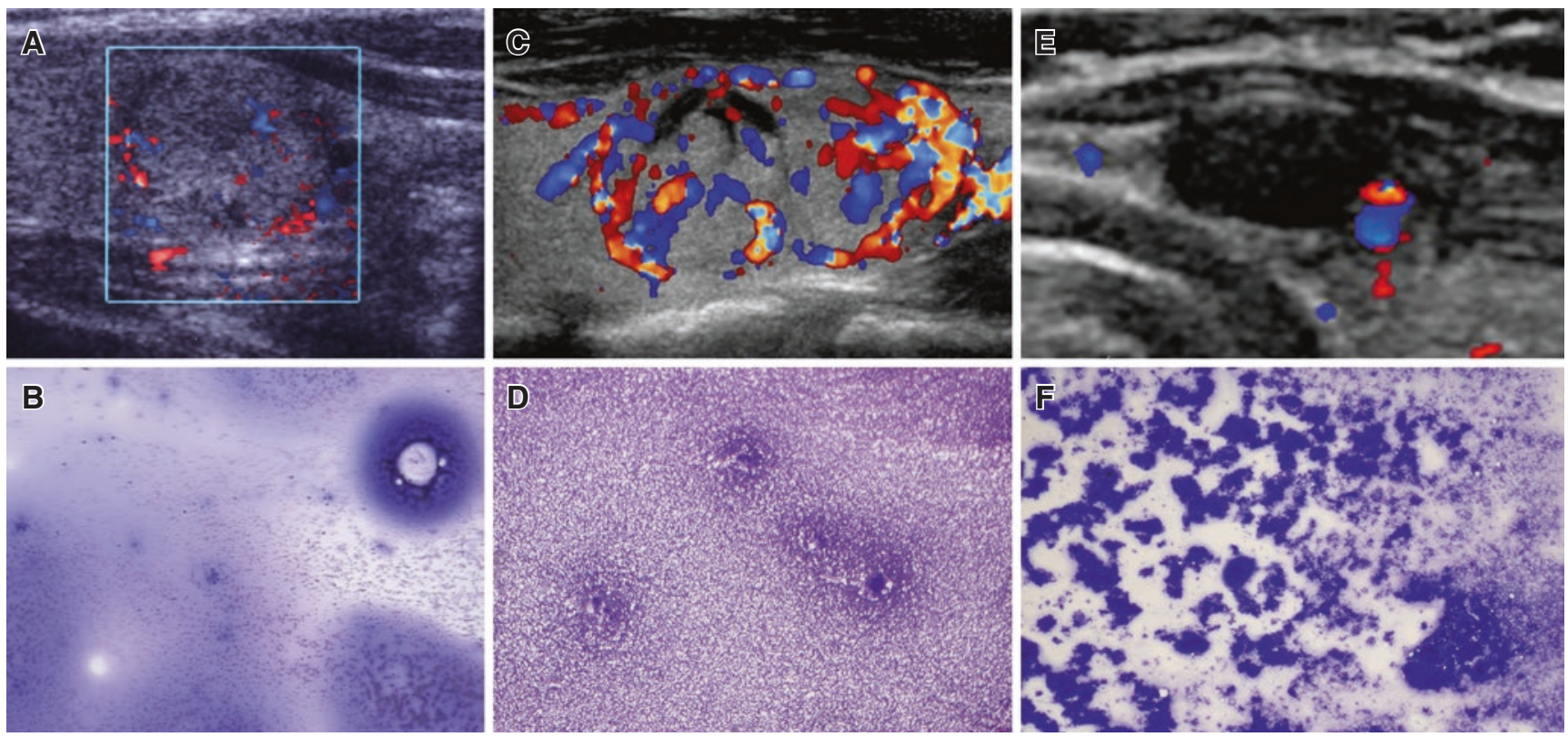

Fig. 20.2 Doppler and Diff-Quik correlation. (A) Isoechoic (same as surrounding thyroid), avascular, nodule with minimal peripheral color flow yielded abundant thin colloid $(\mathbf{B})$; $(\mathbf{C})$ hypervascular nodule with increased color flow (arrow) yielded blood-diluted sample with very low cellularity (D); (E) deeplymarkedly hypoechoic (almost black) avascular nodule with minimal peripheral color flow yielded hypercellular aspirate because no blood was present to dilute the sample $(\mathbf{F})$ (from Yang [12]. All Rights Reserved)

The updated online version of this chapter can be found at https://doi.org/10.1007/978-981-13-1897-9_20 